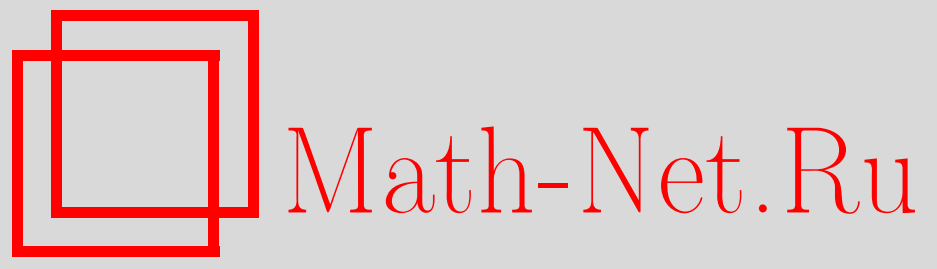

А. Л. Якымив, Распределение длины $m$-го максимального цикла случайной $A$-подстановки, Дискрет. матем., 2005, том 17, выпуск 4, 40-58

DOI: https://doi.org/10.4213/dm128

Использование Общероссийского математического портала Math-Net.Ru подразумевает, что вы прочитали и согласны с пользовательским соглашением http://www . mathnet.ru/rus/agreement

Параметры загрузки:

IP : 54.172 .240 .79

26 апреля 2023 г., 13:24:28

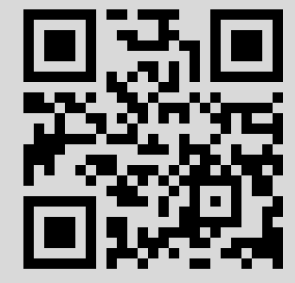




\title{
Распределение длины $m$-го максимального цикла случайной $A$-подстановки
}

\author{
(ㄷ) 2005 г. А. Л. Якымив
}

\begin{abstract}
Пусть $S_{n}$ - симметрическая группа подстановок степени $n, A$ - некоторое подмножество множества натуральных чисел $\mathrm{N}$ и $T_{n}=T_{n}(A)-$ совокупность всех подстановок из $S_{n}$, длины циклов которых принадлежат множеству $A$. Подстановки из $T_{n}$ принято называть $A$-подстановками. Рассматривается широкий класс множеств $A$ асимптотической плотности $\sigma>0$. В статье получены предельные распределения для $\mu_{m}(n) / n$ при $n \rightarrow \infty$ и фиксированном $m \in \mathrm{N}$, где $\mu_{m}(n)$ - длина $m$-го максимального цикла случайной подстановки, равномерно распределенной на $T_{n}$. Показано, что эти предельные распределения совпадают с предельными распределениями соответствующих функционалов от случайных подстановок из $S_{n}$ в известной неравновероятной модели Эвенса с параметром $\sigma$.

Работа выполнена при поддержке Российского фонда фундаментальных исследований, проект 05-01-00583, и программы Президента Российской Федерации поддержки ведущих научных школ, грант НШ-1758.2003.1.
\end{abstract}

\section{1. Введение}

Зафиксируем множество $A \subseteq \mathbf{N}=\{1,2,3, \ldots\}$. $A$-подстановками называются подстановки, длины циклов которых принадлежат множеству $A$ (см. [32]). Пусть $T_{n}=T_{n}(A)$ есть множество всех $A$-подстановок степени $n$. Задав на $T_{n}$ равномерное вероятностное распределение, можно изучать различные функщионалы от случайной подстановки $a_{n}$ из $T_{n}$. Пусть $\zeta_{m n}, m \in A,-$ число циклов подстановки $a_{n}$ длины $m, \zeta_{n}-$ общее число ее циклов, то есть,

$$
\zeta_{n}=\sum_{m \in A} \zeta_{m n}
$$

Всюду через $|X|$ обозначается число элементов конечного множества $X$ и мера Лебега бесконечного множества $X$. Ранее для тех или иных классов множеств $A$ изучались асимптотика $\left|T_{n}\right|$ и предельное поведение (в слабом смысле) $\zeta_{m n}$ и $\zeta_{n}$ при $n \rightarrow \infty$ и фиксированном $m$ (см. $[2,4,6-8,10,12,14,16,17,19-28,37-42])$. Более общие комбинаторные объекты - случайные отображения с ограничениями на длины циклов были рассмотрены в [30-32]. Пусть $S_{n}^{(1)}$ и $S_{n}^{(2)}$ - множества подстановок степени $n$ с нечетными и четными длинами циклов, соответственно. Цикловая структура случайных подстановок с равномерным распределением на некоторых фактор-множествах этих множеств изучалась в $[1,3,34]$. Отметим также, что множество решений уравнения $x^{d}=e$ в симметрической групте $S_{n}$ (e- тождественная подстановка) представляет собой множество 
$A$-подстановок при $A=\left\{d_{0}, d_{1}, \ldots, d_{r}\right\}$, где $1=d_{0}<d_{1}<\ldots<d_{r}=d$ - различные делители числа $d$. Этим, может быть, в частности, объясняется интерес, проявленный со стороны ряда авторов к изучению $A$-подстановок. В [18] имеется специальная глава, в которой изучаются асимптотические свойства решений уравнений, содержаших неизвестную подстановку.

Расположим длины циклов случайной подстановки $a_{n}$ в возрастающем порядке:

$$
l_{n}(1) \leqslant l_{n}(2) \leqslant \ldots \leqslant l_{n}\left(\zeta_{n}\right) .
$$

Мы получили вариационный ряд длин циклов случайной подстановки $a_{n}$. Пусть $\mu_{1}(n)=\mu(n)=l_{n}\left(\zeta_{n}\right)$ и $\mu_{m}(n)=l_{n}\left(\zeta_{n-m+1}\right)$ при $m \in \mathbf{N}$. Случайная величина $\mu(n)$ есть длина максимального цикла подстановки $a_{n}$, а случайная величина $\mu_{m}(n)$ есть длина $m$-го максимального цикла подстановки $a_{n}$ ( $m$-й член справа вариационного ряда (1)). Здесь для широкого класса множеств $A$ асимптотической плотности $\sigma>0$ получены предельные распределения для случайной величины $\mu_{m}(n) / n$ при $n \rightarrow \infty$ и фиксированном $m \in \mathbf{N}$. К настоящему времени этот результат был известен только в случае, когда $\boldsymbol{A}=\mathbf{N}$, то есть, когда вместо $T_{n}$ рассматривается все множество подстановок $S_{n}$.

Экстремальные свойства случайных подстановок, случайных отображений и других комбинаторных структур явились предметом пристального внимания со стороны целого ряда авторов, начиная со знаменитой работы В. Л. Гончарова 1942 г. [9] (история вопроса и подробные ссылки имеются в [13]).

При доказательстве соответствующего утверждения существенно используются идеи упомянутой работы Г. И. Ивченко и Ю. И. Медведева [13], а также статьи автора [38].

\section{2. Основной результат}

Для дальнейшего нам понадобится сформулировать один результат, полученный Г. И. Ивченко и Ю. И. Медведевым в [13]. Зададим на $S_{n}$ распределение вероятностей Эвенса [11] с параметром $\sigma>0$, а именно, каждой подстановке из $S_{n}$ с $k$ циклами припишем вероятность

$$
\frac{\sigma^{k}}{\sigma(\sigma+1) \ldots(\sigma+n-1)}
$$

Соответствующие характеристики случайной подстановки в модели Эвенса будем снабжать индексом $\sigma$. Так, при $m, n \in \mathbf{N}$ случайная величина $\mu_{\sigma m}(n)$ есть длина $m$-го максимального цикла случайной подстановки из $S_{n}$ в модели Эвенса с параметром $\sigma$. Положим при $x>0$ и $m \in \mathbf{N}$

$$
\Phi_{\sigma m}(x)= \begin{cases}1-\sum_{k=m}^{[1 / x]} \frac{(-1)^{k-m} \sigma^{k}}{k(m-1) !(k-m) !} J_{\sigma k}(x) & \text { при } x<m^{-1}, \\ 1 & \text { при } x \geqslant m^{-1},\end{cases}
$$

где

$$
J_{\sigma k}(x)=\int_{V_{k}(x)}\left(1-y_{1}-\ldots-y_{k}\right)^{\sigma-1} \frac{d y_{1} \ldots d y_{k}}{y_{1} \ldots y_{k}}
$$

и

$$
V_{k}(x)=\left\{y=\left(y_{1}, \ldots, y_{k}\right) \in \mathbf{R}^{k}, \quad \sum_{j=1}^{k} y_{j} \leqslant 1, \quad y_{j} \geqslant x, \quad \forall j=1, \ldots, k\right\}
$$


Теорема 1 ([13]). При $n \rightarrow \infty u$ фиксированных $m \in \mathbf{N}, \sigma>0 u x>0$

$$
\mathbf{P}\left\{n^{-1} \mu_{\sigma m}(n) \leqslant x\right\} \rightarrow \Phi_{\sigma m}(x) .
$$

Сформулируем теперь основной результат настоящей статьи.

Теорема 2. Пусть

$$
p(n)=\frac{\left|T_{n}(A)\right|}{n !}=n^{\sigma-1} L(n), \quad n \in \mathbf{N}
$$

где функиия $L(n)$ медленно меняется на бесконечности, $a \sigma \in(0,1]$. Тогда для произвольных фиксированных $m \in N$ и $x>0$ при $n \rightarrow \infty$

$$
\mathbf{P}\left\{n^{-1} \mu_{m}(n) \leqslant x\right\} \rightarrow \Phi_{\sigma m}(x) \text {. }
$$

Замечание 1. Если выполнено (5), то существует предел

$$
\lim _{n \rightarrow \infty} \frac{1}{n}|k: k \in A, k \leqslant n|=\sigma
$$

называемый в теории чисел асимптотической плотностью множества $A$ (см. раздел 3.1 в [29]). Обратное неверно. Например, когда $A$ - множество четных чисел, то $p(n)=0$ для нечетных $n$.

Сравнивая теорему 1 с теоремой 2, мы видим, что предельные распределения в них одни и те же, хотя модели случайных подстановок совершенно разные.

При $A=\mathbf{N}$ из теоремы 2 следует известный результат В. Л. Гончарова [9] $(m=1)$ и результат Л. А. Шеппа и С. П. Ллойда [35] (произвольное $m$ ). Более подробные ссылки и история вопроса даны в [13], см. также монографию В. Ф. Колчина [15], где изложена соответствующая теория экстремумов для подстановок и общих случайных отображений и даны библиографические комментарии к ней до конща 1984 r.

Приведем некоторые примеры, когда выполнено предположение (5) теоремы 2 при $A \neq \mathbf{N}$.

Пример 1. Пусть множество $A$ имеет асимптотическую плотность, равную единице, то есть выполнено соотношение (7) с $\sigma=1$. Тогда справедливо соотношение (5) (см. [37]), а, стало быть, верно утверждение теоремы 2 .

Пример 2. Пусть элементы множества $A$ образуют, начиная с некоторого номера, периодическую последовательность, причем существует $r \in \mathbf{N}$ такое, что для произвольного неотрицательного целого числа $s$ множество $A \cap\{s+1, \ldots, s+r\}$ не может быть вложено ни в какую решетку целых чисел с шагом, большим единицы. Тогда (см. [16, 17]) справедливо соотношение (5).

Пример 3. Пусть функция $f$, определенная на $\mathbf{N}$, удовлетворяет следующим условиям:

(1) $f\left(m_{1} m_{2}\right)=f\left(m_{1}\right) f\left(m_{2}\right)$ при $m_{1}, m_{2} \in \mathbf{N},\left(m_{1}, m_{2}\right)=1$ (здесь и далее $\left(m_{1}, m_{2}\right)$ есть наибольший общий делитель чисел $m_{1}$ и $\left.m_{2}\right), f(1)=1$;

(2) $f$ принимает только значения $-1,0,1$; 
(3) для произвольных $k \in \mathbf{N}, k>1$, функция

$$
\chi(m)=\sum_{d^{k} \mid m} f(d)
$$

принимает только значения 0 и 1.

Пусть $A=A(f, k)$ - множество тех $m \in \mathbf{N}$, для которых $\chi(m)=1$. Тогда (см. [23]) справедливо (5). Далее, в [23] приводятся примеры функций $f$, известных в теории чисел, удовлетворяющих перечисленным требованиям 1-3, таких, как функция Мебиуса, функция Лиувилля.

Пример 4. Пусть $M \in \mathbf{N}, 1 \leqslant i \leqslant M, A_{i}=\left\{m \in \mathbf{N}: m=a_{i} k+b_{i}, k=0,1,2, \ldots\right\}$, где целые $a_{i}>1,1 \leqslant b_{i} \leqslant a_{i}-1,\left(a_{i}, b_{i}\right)=1, A=\bigcup_{i=1}^{M} A_{i}$, прогрессии $A_{i}$ и $A_{j}$ при $i \neq j$ не пересекаются. Тогда (см. [28]) справедливо (5).

Пример 5. Пусть $k_{1}, \ldots, k_{s} \in \mathbf{N}$ таковы, что $k_{i} \geqslant 2, i=1, \ldots, s$ и наибольший общий делитель $\left(k_{i}, k_{j}\right)=1$ при $i \neq j$.

Положим $A=\left\{m \in \mathrm{N}: k_{i} \nmid m, i=1, \ldots, s\right\}$. Тогда (см. [28]) справедливо (5).

Пример 6. Пусть выполнено (7) и для произвольной постоянной $C>1$ при $n \rightarrow \infty$

$$
\frac{1}{n}|k: k \leqslant n, k \in A, m-k \in A| \rightarrow \sigma^{2}
$$

равномерно по $m \in[n, C n]$ (см. [38]). Тогда имеет место соотношение (5).

Пример 7. Пусть множество $A$ случайно, причем случайные величины $\xi_{n}=\chi\{n \in A\}$, $n \in \mathbf{N}$, независимы в совокупности и $p_{n}=\mathbf{P}\left\{\xi_{n}=1\right\} \rightarrow \sigma>0$ при $n \rightarrow \infty$ и $n \in B$ для некоторого множества $B \subseteq N$ асимптотической плотности 1. Тогда соотношения (7) и (8) выполнены для $A$ почти наверное (п.н.) (см. [41]) и, стало быть, при $n \rightarrow \infty$

$$
\mathbf{P}\left\{n^{-1} \mu_{m}(n) \leqslant x \mid A\right\} \rightarrow \Phi_{\sigma m}(x) \text { п.н. }
$$

для произвольных фиксированных $m \in \mathbf{N}$ и $x>0$.

Замечание 2. В [25] по множествам $A$, удовлетворяющим (5), строятся некоторые другие множества, для которых это соотношение выполнено. Например, если для двух непересекающихся множеств $A_{1}, A_{2} \subseteq \mathrm{N}$

$$
p_{i}(n)=\frac{\left|T_{n}\left(A_{i}\right)\right|}{n !}=n^{\sigma_{i}-1} L_{i}(n), \quad n \in \mathbf{N}, \quad i=1,2
$$

где $\sigma_{i}>0$ и функции $L_{i}(n), i=1,2$, медленно меняются на бесконечности, то для множества $A=A_{1} \cup A_{2}$ выполнено (5), причем $\sigma=\sigma_{1}+\sigma_{2}$ и при $n \rightarrow \infty$

$$
L(n) \sim \frac{\Gamma\left(\sigma_{1}\right) \Gamma\left(\sigma_{2}\right)}{\Gamma(\sigma)} L_{1}(n) L_{2}(n) .
$$




\section{3. Доказательства}

Докажем утверждение в замечании 1. Согласно соотношению 6.3 из [4]

$$
\sum_{n=0}^{\infty} p(n) s^{n}=\exp \left(\sum_{n \in A} \frac{s^{n}}{n}\right), \quad s \in[0,1)
$$

(функщи $p(n)$ при $n \in \mathbf{N}$ определена в (5) и дополнительно мы полагаем $p(0)=1)$. Учитывая асимттотику (5), отсюда по абелевой теореме Карамата для степенных рядов (см. теорему 5 в разделе XIII.5 в [36]) получаем, что при $s \uparrow 1$

$$
\exp \left(\sum_{n \in A} \frac{s^{n}}{n}\right) \sim(1-s)^{-\sigma} \Gamma(\sigma) L\left(\frac{1}{1-s}\right)
$$

Дифференцируя (9) по $s$, получаем, что

$$
\sum_{n=1}^{\infty} n p(n) s^{n-1}=\sum_{n \in A} s^{n-1} \exp \left(\sum_{n \in A} \frac{s^{n}}{n}\right), \quad s \in[0,1) .
$$

Применяя к левой части (11) абелеву теорему Карамата и учитывая в правой части (11) асимттотику (10), получим, что при $s \uparrow 1$

$$
s^{-1}(1-s)^{-1-\sigma} \Gamma(1+\sigma) L\left(\frac{1}{1-s}\right) \sim s^{-1} \sum_{n \in A} s^{n}(1-s)^{-\sigma} \Gamma(\sigma) L\left(\frac{1}{1-s}\right),
$$

откуда при $s \uparrow 1$

$$
\sum_{n \in A} s^{n} \sim \sigma(1-s)^{-1}
$$

Из (12) и тауберовой теоремы Карамата следует (7). Замечание доказано.

Ранее было показано, что следствием (5) является существование у множества $A$ логарифмической плотности, равной $\sigma$, то есть существование предела

$$
\lim _{n \rightarrow \infty} \frac{1}{\ln n} \sum_{k \in A, k \leqslant n} \frac{1}{k}=\sigma
$$

(см. следствие 3 в [25]). В замечании 1 содержится более сильное утверждение (как известно, из существования асимптотической плотности множества $A$ следует существование логарифмической плотности $A$, но не наоборот (см. раздел 3.1 в [29])).

Доказательство теоремы 2. Положим при $b \in \mathbf{N}$

$$
\zeta^{b}(n)=\sum_{i>b} \zeta_{i n}
$$

(напомним, что $\zeta_{\text {in }}$ есть число циклов длины $i$ случайной $A$-подстановки степени $n$ ). Ясно, что

$$
\mathbf{P}\left\{\mu_{m}(n) \leqslant b\right\}=\mathbf{P}\left\{\zeta^{b}(n)<m\right\}
$$


Поэтому (см. лемму 2 в [13]), для доказательства теоремы 2 достаточно показать, что для произвольных фиксированных $x, t \in(0,1)$ при $n \rightarrow \infty$ и $b=b(n) \sim n x$

$$
M t^{\zeta^{b}(n)} \rightarrow 1+\sum_{1 \leqslant k<1 / x} \frac{(\sigma(t-1))^{k}}{k !} J_{\sigma k}(x),
$$

где $J_{\sigma k}(x)$ задаются формулой (3). Положим при $t_{1}, \ldots, t_{n} \in[0,1]$

$$
F\left(t_{1}, \ldots, t_{n}\right)=M \prod_{i=1}^{n} t_{i}^{\zeta_{i n}}
$$

Поскольку (см. формулу (0.14) в [32]) для произвольного $s \in[0,1)$ и $t_{1}, \ldots, t_{n} \in[0,1]$

$$
\sum_{n \geqslant 0} p(n) s^{n} F\left(t_{1}, \ldots, t_{n}\right)=\exp \left(a(s)+\sum_{i \in A}\left(t_{i}-1\right) \frac{s^{i}}{i}\right),
$$

где

$$
a(s)=\sum_{i \in A} \frac{s^{i}}{i}
$$

- при $p(n) \neq 0$ справедливо равенство

$$
F\left(t_{1}, \ldots, t_{n}\right)=\frac{1}{p(n)} \operatorname{coef}_{s^{n}} \exp \left(a(s)+\sum_{i \in A}\left(t_{i}-1\right) \frac{s^{i}}{i}\right) .
$$

Стало быть, с учетом (13) и (15),

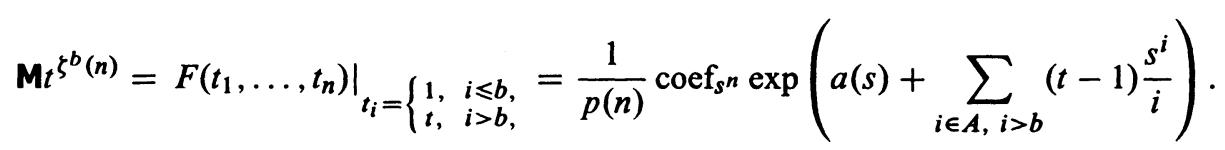

Согласно (9) и (17), $p(l)=\operatorname{coef}_{s} l \exp (a(s))$ при $l \in \mathbf{N} \cup\{0\}$. Поэтому

$$
\begin{aligned}
M t^{\zeta^{b}(n)} & =\frac{1}{p(n)} \operatorname{coef}_{s^{n}} \sum_{l=0}^{\infty} p(l) s^{l} \sum_{k=0}^{\infty} \frac{(t-1)^{k}}{k !}\left(\sum_{i \in A, i>b} \frac{s^{i}}{i}\right)^{k} \\
& =\frac{1}{p(n)} \operatorname{coef}_{s^{n}} \sum_{k=0}^{\infty} \frac{(t-1)^{k}}{k !} \sum_{l=0}^{\infty} p(l) s^{l}\left(\sum_{i \in A, i>b} \frac{s^{i}}{i}\right)^{k} \\
& =1+\sum_{1 \leqslant k<n / b} \frac{(t-1)^{k}}{k !} S_{k}(n),
\end{aligned}
$$

где

$$
S_{k}(n)=\sum_{r=(b+1) k}^{n} \frac{p(n-r)}{p(n)} \sum \frac{1}{i_{1} \ldots i_{k}}
$$


(суммирование в последней сумме ведется по тем $\left(i_{1}, \ldots, i_{k}\right) \in \mathrm{N}^{k}$, для которых $i_{1}+\ldots+i_{k}=r, i_{j}>b, i_{j} \in A$ при $\left.j=1, \ldots, k\right)$. Стало быть,

$$
S_{k}(n)=\sum_{\left(i_{1}, \ldots, i_{k}\right) \in A_{k}(n)} \frac{1}{i_{1} \ldots i_{k}} \frac{p\left(n-i_{1}-\ldots-i_{k}\right)}{p(n)},
$$

где

$$
A_{k}(n)=\left\{\left(i_{1}, \ldots, i_{k}\right) \in \mathbf{N}^{k}, \quad \sum_{j=1}^{k} i_{j} \leqslant n, \quad i_{j}>b, \quad i_{j} \in A, \quad j=1, \ldots, k\right\} .
$$

Для функщии $L(n)$, определенной в равенстве (5), согласно лемме 6 на с.462 в [42] подберем такую последовательность натуральных чисел $a(n)$, что $a(n) \rightarrow \infty, a(n)=o(n)$ при $n \rightarrow \infty$ и

$$
\frac{L(r)}{L(n)} \rightarrow 1
$$

при $n \rightarrow \infty$ равномерно по $r \in[a(n), n]$. Зафиксируем произвольное $\varepsilon \in[0, \sigma]$. Поскольку функщия $p(n)$ правильно меняется на бесконечности с показателем $\sigma-1$ (см. (5)), согласно лемме 2.1 из [5] существует $n_{0}=n_{0}(\varepsilon) \in \mathbf{N}$ такое, что для любых $n \geqslant n_{0}$ и $r \in\left[n_{0}, n(1-\varepsilon)\right]$

$$
\frac{p(r)}{p(n)} \leqslant\left(\frac{r}{n}\right)^{\sigma-1-\varepsilon}
$$

Без ограничения общности считаем, что при $n \geqslant n_{0}$

$$
a(n)<n(1-\varepsilon) .
$$

Представим $S_{k}(n)$ в виде суммы

$$
\begin{aligned}
S_{k}(n) & =S_{k}^{(1)}(n)+S_{k}^{(2)}(n)+S_{k}^{(3)}(n) \\
& =\sum_{l=1}^{3} \sum_{\left(i_{1}, \ldots, i_{k}\right) \in A_{k}^{(l)}(n)} \frac{1}{i_{1} \ldots i_{k}} \frac{p\left(n-i_{1}-\ldots-i_{k}\right)}{p(n)},
\end{aligned}
$$

где $A_{k}^{(1)}(n), A_{k}^{(2)}(n)$ и $A_{k}^{(3)}(n)$ - подмножества $A_{k}(n)$, отвечающие неравенствам

$$
\sum_{j=1}^{k} i_{j} \leqslant n-a(n), \quad n-a(n)<\sum_{j=1}^{k} i_{j} \leqslant n-n_{0}, \quad n-n_{0}<\sum_{j=1}^{k} i_{j} \leqslant n,
$$

соответственно. В силу (5) и (20) при $n \rightarrow \infty$

$$
\begin{aligned}
S_{k}^{(1)}(n) & =\sum_{\left(i_{1}, \ldots, i_{k}\right) \in A_{k}^{(1)}(n)} \frac{1}{i_{1} \ldots i_{k}} \frac{p\left(n-i_{1}-\ldots-i_{k}\right)}{p(n)} \\
& =(1+o(1)) \sum_{\left(i_{1}, \ldots, i_{k}\right) \in A_{k}^{(1)}(n)} \frac{1}{i_{1} \ldots i_{k}}\left(1-\frac{i_{1}+\ldots+i_{k}}{n}\right)^{\sigma-1} .
\end{aligned}
$$


Согласно (21) и (22) при $n \geqslant n_{0}$

$$
\begin{aligned}
S_{k}^{(2)}(n) & =\sum_{\left(i_{1}, \ldots, i_{k}\right) \in A_{k}^{(2)}(n)} \frac{1}{i_{1} \ldots i_{k}} \frac{p\left(n-i_{1}-\ldots-i_{k}\right)}{p(n)} \\
& \leqslant \sum_{\left(i_{1}, \ldots, i_{k}\right) \in A_{k}^{(2)}(n)} \frac{1}{i_{1} \ldots i_{k}}\left(1-\frac{i_{1}+\ldots+i_{k}}{n}\right)^{\rho-1},
\end{aligned}
$$

где $\rho=\sigma-\varepsilon>0$. Для произвольного $j=1, \ldots, k$ справедливо неравенство $i_{j}>b$, поэтому

$$
\begin{aligned}
S_{k}^{(2)}(n) & \leqslant \frac{1}{b^{k}} \sum_{\left(i_{1}, \ldots, i_{k}\right) \in A_{k}^{(2)}(n)}\left(1-\frac{i_{1}+\ldots+i_{k}}{n}\right)^{\rho-1} \\
& \leqslant C n^{-k} \sum_{\left(i_{1}, \ldots, i_{k}\right) \in A_{k}^{(2)}(n)}\left(1-\frac{i_{1}+\ldots+i_{k}}{n}\right)^{\rho-1}
\end{aligned}
$$

для достаточно больших $n \geqslant n_{1} \geqslant n_{0}$, так как $b=b(n) \sim x n$ при $n \rightarrow \infty$. При $r \in\left[n_{0}, a(n)\right]$ положим

$$
A_{k}^{(2)}(n, r)=\left\{\left(i_{1}, \ldots, i_{k}\right) \in A_{k}^{(2)}(n), \quad \sum_{j=1}^{k} i_{j}=n-r\right\} .
$$

Из неравенства (26) при $n \geqslant n_{1}$ следует, что

$$
S_{k}^{(2)}(n) \leqslant C n^{-k} \sum_{r=n_{0}}^{a(n)} \sum_{\left(i_{1}, \ldots, i_{k}\right) \in A_{k}^{(2)}(n, r)}\left(\frac{r}{n}\right)^{\rho-1}=c n^{-k+1-\rho} \sum_{r=n_{0}}^{a(n)} r^{\rho-1}\left|A_{k}^{(2)}(n, r)\right| .
$$

Заметим, что

$$
\left|A_{k}^{(2)}(n, r)\right| \leqslant\left|\left(i_{1}, \ldots, i_{k}\right) \in \mathbf{N}^{k} \cap[1, n]^{k}, \sum_{j=1}^{k} i_{j}=n-r\right| \leqslant n^{k-1} .
$$

Поэтому при $n \geqslant n_{0}$

$$
S_{k}^{(2)}(n) \leqslant C n^{-\rho} \sum_{r=n_{0}}^{a(n)} r^{\rho-1}
$$

откуда при $n \rightarrow \infty$

$$
S_{k}^{(2)}(n)=O\left(\left(\frac{a(n)}{n}\right)^{\rho}\right)=o(1)
$$

так как $\rho>0$. Далее, поскольку $p(l) \leqslant 1$ при $l \geqslant 0$, при $n \geqslant n_{1}$ справедливы соотношения

$$
\begin{aligned}
S_{k}^{(3)}(n) & =\sum_{\left(i_{1}, \ldots, i_{k}\right) \in A_{k}^{(3)}(n)} \frac{1}{i_{1} \ldots i_{k}} \frac{p\left(n-i_{1}-\ldots-i_{k}\right)}{p(n)} \leqslant \frac{1}{p(n)} \sum_{\left(i_{1}, \ldots, i_{k}\right) \in A_{k}^{(3)}(n)} \frac{1}{i_{1} \ldots i_{k}} \\
& \leqslant \frac{C n^{-k}}{p(n)}\left|A_{k}^{(3)}(n)\right| \leqslant \frac{C}{p(n) n^{k}} n_{0} n^{k-1}=\frac{C n_{0}}{n p(n)} .
\end{aligned}
$$


Так как согласно (5) $n p(n)=n^{\sigma} L(n) \rightarrow \infty$ при $n \rightarrow \infty$, из полученной оценки следует, что при $n \rightarrow \infty$

$$
S_{k}^{(3)}(n)=o(1)
$$

Из соотношений (23), (24), (27) и (28) следует, что при $n \rightarrow \infty$

$$
S_{k}(n)=(1+o(1)) I_{k}(n)+o(1),
$$

где

$$
I_{k}(n)=\sum_{\left(i_{1}, \ldots, i_{k}\right) \in A_{k}^{(1)}(n)} \frac{1}{i_{1} \ldots i_{k}}\left(1-\frac{i_{1}+\ldots+i_{k}}{n}\right)^{\sigma-1}
$$

Далее при $u, v \in \mathbf{R}^{k}$ соотношения $u<v(u \leqslant v)$ означаюя соответствующие покомпонентные неравенства, при $u<v$

$$
[u, v)=\left\{y \in \mathbf{R}^{k}, u \leqslant y<v\right\}, \quad[u, v]=\left\{y \in \mathbf{R}^{k}, u \leqslant y \leqslant v\right\}
$$

- соответствуюшие $k$-мерные кубы. Пусть $e=(1, \ldots, 1)-k$-мерный вектор,

$$
\begin{gathered}
B_{k}(n)=\bigcup_{i \in A_{k}^{(1)}(n)}(i+[0, e)), \\
I_{k}^{\prime}(n)=\int_{B_{k}(n)} f_{k n}(y) d y,
\end{gathered}
$$

где

$$
f_{k n}(y)=\frac{1}{y_{1} \ldots y_{k}}\left(1-\frac{y_{1}+\ldots+y_{k}}{n}\right)^{\sigma-1}
$$

Покажем, что при $n \rightarrow \infty$

$$
I_{k}(n)=I_{k}^{\prime}(n)+o(1) .
$$

В самом деле,

$$
\begin{aligned}
\left|I_{k}(n)-I_{k}^{\prime}(n)\right| & \leqslant \sum_{i \in A_{k}^{(1)}(n)}\left|\int_{i+[0, e)} f_{k n}(y) d y-f_{k n}(i)\right| \\
& =\sum_{i \in A_{k}^{(1)}(n)}\left|\int_{i+[0, e)}\left(f_{k n}(y)-f_{k n}(i)\right) d y\right| \\
& \leqslant \sum_{i \in A_{k}^{(1)}(n)} \int_{i+[0, e)}\left|f_{k n}^{\prime}(y)\right||y-i| d y \\
& \leqslant \sqrt{k} \sum_{i \in A_{k}^{(1)}(n)} \max _{y \in i+[0, e]}\left|f_{k n}^{\prime}(y)\right| .
\end{aligned}
$$


При $l=1, \ldots, k$

$$
\begin{aligned}
\frac{\partial f_{k n}(y)}{\partial y_{l}} & =\frac{\partial}{\partial y_{l}}\left(\frac{1}{y_{1} \ldots y_{k}}\left(1-\frac{y_{1}+\ldots+y_{k}}{n}\right)^{\sigma-1}\right) \\
& =-y_{l}^{-1} f_{k n}(y)-\frac{(\sigma-1)}{n} f_{k n}(y)\left(1-\frac{y_{1}+\ldots+y_{k}}{n}\right)^{-1} \\
& =f_{k n}(y)\left(\frac{(1-\sigma)}{n}\left(1-\frac{y_{1}+\ldots+y_{k}}{n}\right)^{-1}-y_{l}^{-1}\right) .
\end{aligned}
$$

Далее,

$$
\begin{aligned}
& \frac{1}{n} \sum_{i \in A_{k}^{(1)}(n)} \max _{y \in i+[0, e]}\left(f_{k n}(y)\left(1-\frac{y_{1}+\ldots+y_{k}}{n}\right)^{-1}\right) \\
& \leqslant \frac{1}{n} \sum_{i \in A_{k}^{(1)}(n)} \frac{1}{i_{1} \ldots i_{k}}\left(1-\frac{i_{1}+\ldots+i_{k}+k}{n}\right)^{\sigma-2} \\
& \leqslant C n^{-k} n^{-1}\left(1-\frac{n-a(n)+k}{n}\right)^{\sigma-2}\left|A_{k}^{(1)}(n)\right| \\
& \leqslant \frac{C}{n^{k+1}}\left(\frac{a(n)-k}{n}\right)^{\sigma-2} n^{k}=C n^{-1}\left(\frac{a(n)-k}{n}\right)^{\sigma-2} .
\end{aligned}
$$

Без ограничения общности мы можем считать, что $a(n) \geqslant \sqrt{n}+k$. Тогда

$$
\begin{aligned}
\frac{1}{n} \sum_{i \in A_{k}^{(1)}(n)} \max _{y \in i+[0, e]}\left(f_{k n}(y)\left(1-\frac{y_{1}+\ldots+y_{k}}{n}\right)^{-1}\right) & \leqslant C n^{-1}\left(n^{-1 / 2}\right)^{\sigma-2} \\
& =C n^{-1} n^{1-\sigma / 2}=o(1)
\end{aligned}
$$

при $n \rightarrow \infty$. Для произвольного $l=1, \ldots, k$

$$
\begin{aligned}
\sum_{i \in A_{k}^{(1)}(n)} \max _{y \in i+[0, e]}\left(\frac{f_{k n}(y)}{y_{l}}\right) & \leqslant \sum_{i \in A_{k}^{(1)}(n)} \frac{1}{y_{l}} \frac{1}{i_{1} \ldots i_{k}}\left(1-\frac{i_{1}+\ldots+i_{k}+k}{n}\right)^{\sigma-1} \\
& \leqslant C n^{-k-1}\left(\frac{a(n)-k}{n}\right)^{\sigma-1}\left|A_{k}^{(1)}(n)\right| \\
& \leqslant C n^{-k-1}\left(n^{-1 / 2}\right)^{\sigma-1} n^{k}=C n^{-1} n^{(1-\sigma) / 2}=C n^{-(\sigma+1) / 2},
\end{aligned}
$$

где $C n^{-(\sigma+1) / 2} \rightarrow 0$ при $n \rightarrow \infty$. Из соотношений (35)-(38) следует (34). Из (34) и (29) получаем, что при $n \rightarrow \infty$

$$
S_{k}(n)=(1+o(1)) I_{k}^{\prime}(n)+o(1)
$$

где $I_{k}^{\prime}(n)$ задается формулой (32). Положим

$$
B_{k}^{\prime}(n)=B_{k}(n) / n, \quad f_{k}(y)=f_{k 1}(y),
$$


где $f_{k n}(y)$ задается формулой (33). Заметим, что

$$
I_{k}^{\prime}(n)=\int_{B_{k}^{\prime}(n)} f_{k}(z) d z
$$

Согласно (18), $k<n / b=1 / x+o(1)$ при $n \rightarrow \infty$. Поэтому можно считать, что $k<1 / x$. Возьмем произвольные $u, v \in \mathbf{R}^{k}, u<v$, причем $u_{j} \geqslant x$ для всех $j=1, \ldots, k$ и $\sum_{j=1}^{k} v_{j}<1$ (это возможно, так как $k<1 / x$ ). Справедливо неравенство

$$
\int_{[u, v) \cap B_{k}^{\prime}(n)} f_{k}(y) d y \leqslant \max _{y \in[u, v]} f_{k}(y)\left|[u, v] \cap B_{k}^{\prime}(n)\right| .
$$

В свою очередь,

$$
\begin{aligned}
\left|[u, v] \cap B_{k}^{\prime}(n)\right| & =\sum_{i \in A_{k}^{(1)}(n)}\left|\left(\frac{i}{n}+\left[0, \frac{e}{n}\right)\right) \cap[u, v]\right| \\
& =\sum_{i \in A_{k}^{(1)}(n)} n^{-k}|(i+[0, e)) \cap[n u, n v]|=\Sigma_{1}+\Sigma_{2}
\end{aligned}
$$

причем

$$
\Sigma_{1}=n^{-k} \sum_{i \in A_{k}^{(1)}(n)} \chi\{i \geqslant n u, i+e \leqslant n v\}
$$

и

$$
0 \leqslant \Sigma_{2} \leqslant n^{-k} \sum_{i \in A_{k}^{(1)}(n)} \chi\left\{\exists l=1, \ldots, k: i_{l}+1>n v_{l}, i \in[n u, n v]\right\}
$$

Ясно, что

$$
\begin{aligned}
\Sigma_{1} & =n^{-k}\left|i \in A_{k}^{(1)}(n): i \geqslant n u, i+e \leqslant n v\right| \\
& =n^{-k}\left|i \in \mathrm{N}^{k}: \sum_{j=1}^{k} i_{j} \leqslant n-a(n), i_{j}>b, i_{j} \geqslant n u_{j}, i_{j}+1 \leqslant n v_{j}, i_{j} \in A, j=1, \ldots, k\right| \\
& =n^{-k}\left|i \in \mathrm{N}^{k}: \sum_{j=1}^{k} i_{j} \leqslant n-a(n), \max \left(b+1, n u_{j}\right) \leqslant i_{j} \leqslant n v_{j}-1, i_{j} \in A, j=1, \ldots, k\right| .
\end{aligned}
$$

Если $i_{j} \leqslant n v_{j}-1$ для всех $j=1, \ldots, k$, то

$$
\sum_{j=1}^{k} i_{j} \leqslant n \sum_{j=1}^{k} v_{j}-k \leqslant n-a(n)
$$

при достаточно больших $n \geqslant n_{2} \geqslant n_{1}$, так как $\sum_{j=1}^{k} v_{j}<1$, а $a(n)=o(n)$ при $n \rightarrow \infty$.

Поэтому при $n \geqslant n_{2}$

$$
\begin{aligned}
\Sigma_{1} & =n^{-k}\left|i \in \mathbf{N}^{k}: \max \left(b+1, n u_{j}\right) \leqslant i_{j} \leqslant n v_{j}-1, i_{j} \in A \forall j=1, \ldots, k\right| \\
& =n^{-k}\left|i \in \mathbf{N}^{k}: i_{j} \in\left[\max \left(b+1, n u_{j}\right), n v_{j}-1\right] \cap A \forall j=1, \ldots, k\right| \\
& =n^{-k} \prod_{j=1}^{k}\left|l: l \in N, l \in\left[\max \left(b+1, n u_{j}\right), n v_{j}-1\right] \cap A\right| .
\end{aligned}
$$


Так как асимптотическая плотность множества $A$ равна $\sigma$ (см. (7)) и

$$
\max \left(b+1, n u_{j}\right) \sim n u_{j}, \quad u_{j} \geqslant x, \quad b \sim n x
$$

при $n \rightarrow \infty$, нетрудно видеть, что при $n \rightarrow \infty$

$$
\left|l: l \in \mathbf{N}, l \in\left[\max \left(b+1, n u_{j}\right), n v_{j}-1\right] \cap A\right|=(1+o(1))\left(v_{j}-u_{j}\right) \sigma n .
$$

Поэтому при $n \rightarrow \infty$

$$
\Sigma_{1}=(1+o(1)) \sigma^{k}|[u, v]|
$$

Далее, согласно (44),

$$
\begin{aligned}
0 \leqslant \Sigma_{2} & \leqslant n^{-k} \sum_{i \in(N \cap[1, n])^{k}} \chi\left\{\exists l=1, \ldots, k: i_{l}+1>n v_{l}, i \in[n u, n v]\right\} \\
& =n^{-k}\left|i \in(\mathrm{N} \cap[1, n])^{k}: \exists l=1, \ldots, k\right| i_{l}+1>n v_{l}, i \in[n u, n v] \mid \\
& \leqslant n^{-k}\left|i \in(\mathrm{N} \cap[1, n])^{k}: \exists l=1, \ldots, k\right| i_{l}=\left[n v_{l}\right], i \in[n u, n v] \mid \\
& \leqslant n^{-k} \sum_{l=1}^{k}\left|i \in(N \cap[1, n])^{k}, i_{l}=\left[n v_{l}\right]\right| \leqslant n^{-k} \sum_{l=1}^{k} n^{k-1}=k n^{-1}
\end{aligned}
$$

где последний член стремится к нулю при $n \rightarrow \infty$. Из (41), (42), (45) и (46) следует, что

$$
\limsup _{n \rightarrow \infty} \int_{[u, v) \cap B_{k}^{\prime}(n)} f_{k}(y) d y \leqslant \max _{y \in[u, v]} f_{k}(y) \sigma^{k} \|[u, v] \mid .
$$

Совершенно аналогично получаем, что

$$
\liminf _{n \rightarrow \infty} \int_{[u, v) \cap B_{k}^{\prime}(n)} f_{k}(y) d y \geqslant \min _{y \in[u, v]} f_{k}(y) \sigma^{k}|[u, v]| .
$$

Положим

$$
f_{k}^{(n)}(y)= \begin{cases}f_{k}(y), & y \in B_{k}^{\prime}(n) \\ 0, & y \notin B_{k}^{\prime}(n)\end{cases}
$$

Из (47) и (48) следует, что при $x e \leqslant u<v$ и $\sum_{j=1}^{k} v_{j}<1$

$$
\limsup _{n \rightarrow \infty} \int_{[u, v)} f_{k}^{(n)}(y) d y \leqslant \max _{y \in[u, v]} f_{k}(y) \sigma^{k}|[u, v]|
$$

и

$$
\liminf _{n \rightarrow \infty} \int_{[u, v)} f_{k}^{(n)}(y) d y \geqslant \min _{y \in[u, v]} f_{k}(y) \sigma^{k}|[u, v]| .
$$

Введем обозначения

$$
\begin{aligned}
U_{k}(n) & =\left\{i \in \mathrm{N}^{k}: \sum_{j=1}^{k} i_{j} \leqslant n-a(n), i>b e\right\}, \\
V_{k n} & =\bigcup_{i \in U_{k}(n)}\left(\frac{i}{n}+\frac{[0, e)}{n}\right) .
\end{aligned}
$$


Напомним, что, как и в (4),

$$
V_{k}=V_{k}(x)=\left\{y \in \mathbf{R}^{k}: y \geqslant x e, \sum_{j=1}^{k} y_{j} \leqslant 1\right\}
$$

Согласно определению $U_{k}(n)$ и $A_{k}^{(1)}(n)$,

$$
A_{k}^{(1)}(n)=U_{k}(n) \cap A .
$$

Так как

$$
B_{k}^{\prime}(n)=\bigcup_{i \in A_{k}^{(1)}(n)}\left(\frac{i}{n}+\frac{[0, e)}{n}\right) \subseteq V_{k n}
$$

и функция $f_{k}^{(n)}(y)$ равна нулю вне $B_{k}^{\prime}(n)$, в силу (40) и определения функции $f_{k}^{(n)}(y)$ справедливо равенство

$$
I_{k}^{\prime}(n)=\int_{B_{k}^{\prime}(n)} f_{k}(y) d y=\int_{B_{k}^{\prime}(n)} f_{k}^{(n)}(y) d y=\int_{V_{k n}} f_{k}^{(n)}(y) d y .
$$

Далее,

$$
\begin{aligned}
I_{k}^{\prime}(n) & =\int_{V_{k}} f_{k}^{(n)}(y) d y+\int_{V_{k n} \backslash V_{k}} f_{k}^{(n)}(y) d y-\int_{V_{k} \backslash V_{k n}} f_{k}^{(n)}(y) d y \\
& =\int_{V_{k}} f_{k}^{(n)}(y) d y+\int_{V_{k n} \backslash V_{k}} f_{k}^{(n)}(y) d y
\end{aligned}
$$

так как $f_{k}^{(n)}(y)=0$ при $y \in V_{k} \backslash V_{k n}\left(B_{k}^{\prime}(n) \subseteq V_{k n}\right)$. Покажем, что при $n \rightarrow \infty$

$$
\left|V_{k n} \backslash V_{k}\right| \rightarrow 0
$$

В самом деле, если $y \in V_{k n} \backslash V_{k}$, то $y=i / n+y_{0}$, где $y_{0} \in[0, e / n)$ и $i \in U_{k}(n)$, причем

$$
\sum_{j=1}^{k} y_{j}=\frac{1}{n} \sum_{j=1}^{k} i_{j}+\sum_{j=1}^{k} y_{0 j} \leqslant 1-\frac{a(n)}{n}+\frac{k}{n}=1-\frac{a(n)-k}{n} \leqslant 1,
$$

так как $a(n) \geqslant k$ при $n \in \mathbf{N}$. Поэтому существует $j=1, \ldots, k$ такое, что $y_{j}<x$. Так как

$$
\frac{i_{j}}{n}+y_{0 j}<x, \quad \frac{i_{j}}{n}>\frac{b}{n}, \quad \frac{b}{n}<\frac{i_{j}}{n}<x-y_{0 j} \leqslant x,
$$

получаем, что $i_{j} \in(b, n x)$. Стало быть,

$$
\begin{aligned}
\left|V_{k n} \backslash V_{k}\right| & \leqslant n^{-k}\left|i \in U_{k}(n): \exists j=1, \ldots, k\right| i_{j} \in(b, n x) \mid \\
& \leqslant n^{-k} \sum_{j=1}^{k}\left|i \in U_{k}(n): i_{j} \in(b, n x)\right| \leqslant n^{-k} \sum_{j=1}^{k}\left|i \in(\mathrm{N} \cap[1, n])^{k}: i_{j} \in(b, n x)\right| \\
& \leqslant n^{-k} k n^{k-1}(|b-n x|+1)=k\left|\frac{b}{n}-x\right|+\frac{1}{n}
\end{aligned}
$$


где последний член стремится к нулю при $n \rightarrow \infty$, так как $b / n \rightarrow x$ при $n \rightarrow \infty$. Таким образом, (53) выполнено. Поэтому

$$
0 \leqslant \int_{V_{k n} \backslash V_{k}} f_{k}^{(n)}(y) d y \leqslant \int_{V_{k n} \backslash V_{k}} f_{k}(y) d y
$$

и правая часть стремится к нулю при $\boldsymbol{n} \rightarrow \infty$.

Из (52) и последнего соотношения следует, что при $n \rightarrow \infty$

$$
I_{k}^{\prime}(n)=\int_{V_{k}} f_{k}^{(n)}(y) d y+o(1)
$$

Стало быть, согласно (29), (34) и (54), при $n \rightarrow \infty$

$$
S_{k}(n)=(1+o(1)) I_{k}^{\prime \prime}(n)+o(1),
$$

где

$$
I_{k}^{\prime \prime}(n)=\int_{V_{k}} f_{k}^{(n)}(y) d y .
$$

При $\delta \in(0,1-k x)$ и $\theta \in(0,(1-k x-\delta) / k)$ положим

$$
\begin{aligned}
V_{k}^{\delta} & =\left\{y \in \mathbf{R}^{k}: \sum_{j=1}^{k} y_{j} \leqslant 1-\delta, y_{j} \geqslant x j=1, \ldots, k\right\}, \\
T_{k}(\delta, \theta) & =\left\{i \in \mathbf{N}^{k}: \theta \sum_{j=1}^{k} i_{j}+\theta k \leqslant 1-\delta, \theta i_{j} \geqslant x j=1, \ldots, k\right\}, \\
V_{k}^{\delta, \theta} & =\bigcup_{i \in T_{k}(\delta, \theta)} \theta(i+[0, e))=\bigcup_{i \in T_{k}(\delta, \theta)} \Delta_{i},
\end{aligned}
$$

где $\Delta_{i}=\theta(i+[0, e))$. Нетрудно заметить, что при указанных условиях на $\delta$ и $\theta$ множества $V_{k}^{\delta}$ и $V_{k}^{\delta, \theta}$ имеют положительную меру Лебега, а множество $T_{k}(\delta, \theta)$ непусто. Применяя неравенства (49) и (50) к каждому кубу $\Delta_{i}, i \in T_{k}(\delta, \theta)$, получаем, что

$$
\begin{aligned}
& \liminf _{n \rightarrow \infty} \int_{V_{k}^{\delta, \theta}} f_{k}^{(n)}(y) d y \geqslant \sigma^{k} \sum_{i \in T_{k}(\delta, \theta)} \min _{y \in \bar{\Delta}_{i}} f_{k}(y)\left|\Delta_{i}\right|, \\
& \limsup _{n \rightarrow \infty} \int_{V_{k}^{\delta, \theta}} f_{k}^{(n)}(y) d y \leqslant \sigma^{k} \sum_{i \in T_{k}(\delta, \theta)} \max _{y \in \bar{\Delta}_{i}} f_{k}(y)\left|\Delta_{i}\right|
\end{aligned}
$$

(здесь и далее $\bar{H}$ обозначает замыкание множества $H \subseteq \mathbf{R}^{k}$ ). Отсюда, с учетом неравенства $f_{k}^{(n)}(y) \leqslant f_{k}(y)$ и включений $V_{k}^{\delta, \theta} \subseteq V_{k}^{\delta} \subseteq V_{k}$, получаем, что

$$
\begin{aligned}
& \liminf _{n \rightarrow \infty} \int_{V_{k}} f_{k}^{(n)}(y) d y \geqslant \sigma^{k} \sum_{i \in T_{k}(\delta, \theta)} \min _{y \in \bar{\Delta}_{i}} f_{k}(y)\left|\Delta_{i}\right|, \\
& \limsup _{n \rightarrow \infty} \int_{V_{k}} f_{k}^{(n)}(y) d y \leqslant \sigma^{k} \sum_{i \in T_{k}(\delta, \theta)} \max _{y \in \bar{\Delta}_{i}} f_{k}(y)\left|\Delta_{i}\right|+\int_{V_{k} \backslash V_{k}^{\delta, \theta}} f_{k}(y) d y .
\end{aligned}
$$


Докажем, что при $\theta \rightarrow 0$

$$
\begin{aligned}
& \left|V_{k}^{\delta} \backslash V_{k}^{\delta, \theta}\right| \rightarrow 0, \\
& \Sigma_{1}(\delta, \theta)=\sum_{i \in T_{k}(\delta, \theta)} \max _{y \in \bar{\Delta}_{i}} f_{k}(y)\left|\Delta_{i}\right| \rightarrow \int_{V_{k}^{\delta}} f_{k}(y) d y \text {, } \\
& \Sigma_{2}(\delta, \theta)=\sum_{i \in T_{k}(\delta, \theta)} \min _{y \in \bar{\Delta}_{i}} f_{k}(y)\left|\Delta_{i}\right| \rightarrow \int_{V_{k}^{\delta}} f_{k}(y) d y .
\end{aligned}
$$

Рассмотрим

$$
T_{k}^{\prime}(\delta, \theta)=\left\{i \in \mathbf{N}^{k}: \theta \sum_{j=1}^{k} i_{j} \leqslant 1-\delta, \theta \sum_{j=1}^{k} i_{j}+\theta k>1-\delta, \theta i_{j} \geqslant x, j=1, \ldots, k\right\},
$$

и срезанные кубы

$$
\Delta_{i}=\theta(i+[0, e)) \cap V_{k}^{\delta}, \quad i \in T_{k}^{\prime}(\delta, \theta) .
$$

Пусть

$$
\begin{aligned}
T_{k}^{\prime \prime}(\delta, \theta) & =T_{k}(\delta, \theta) \cup T_{k}^{\prime}(\delta, \theta), \\
\Sigma_{\max }(\delta, \theta) & =\Sigma_{1}(\delta, \theta)+\sum_{i \in T_{k}^{\prime}(\delta, \theta)} \max _{y \in \bar{\Delta}_{i}} f_{k}(y)\left|\Delta_{i}\right|
\end{aligned}
$$

Сумма $\Sigma_{\max }(\delta, \theta)$ есть верхняя сумма Дарбу для $k$-мерного интеграла Римана функции $f_{k}$ по выбранному нами разбиению $\Delta_{i}, i \in T_{k}^{\prime \prime}(\delta, \theta)$ множества $V_{k}^{\delta}$. Поскольку функция $f_{k}$ непрерывна на множестве $V_{k}^{\delta}$, мы видим, что при $\theta \rightarrow 0$

$$
\Sigma_{\max }(\delta, \theta) \rightarrow \int_{V_{k}^{\delta}} f_{k}(y) d y
$$

Ясно, что

$$
\left|V_{k}^{\delta} \backslash V_{k}^{\delta, \theta}\right|=\sum_{i \in T_{k}^{\prime}(\delta, \theta)}\left|\Delta_{i}\right| \leqslant \theta^{k} \sum_{i \in T_{k}^{\prime}(\delta, \theta)} 1=\theta^{k}\left|T_{k}^{\prime}(\delta, \theta)\right| .
$$

Заметим, что при $i \in T_{k}^{\prime}(\delta, \theta)$

$$
\sum_{j=1}^{k} i_{j} \leqslant \frac{1-\delta}{\theta}, \quad \sum_{j=1}^{k} i_{j}+k>\frac{1-\delta}{\theta}
$$

или

$$
\sum_{j=1}^{k} i_{j} \in\left(\frac{1-\delta}{\theta}-k, \frac{1-\delta}{\theta}\right]
$$

На полуинтервале $D=((1-\delta) / \theta-k,(1-\delta) / \theta]$ не более $k$ целых точек, поэтому

$$
\left|T_{k}^{\prime}(\delta, \theta)\right| \leqslant\left|i \in\left(\left(1, \theta^{-1}\right) \cap \mathbf{N}\right)^{k}, \sum_{j=1}^{k} i_{j} \in D\right| \leqslant \frac{k}{\theta^{k-1}} .
$$


Иными словами,

$$
\left|V_{k}^{\delta} \backslash V_{k}^{\delta, \theta}\right| \leqslant \frac{\theta^{k}}{\theta^{k-1}} k=\theta k
$$

и правая часть этого соотношения стремится к нулю при $\theta \rightarrow 0$.

Из последнего соотношения следует (59). Далее, в силу (59),

$$
\sum_{i \in T_{k}^{\prime}(\delta, \theta)} \max _{y \in \bar{\Delta}_{i}} f_{k}(y)\left|\Delta_{i}\right| \leqslant \max _{y \in V_{k}^{\delta}} f_{k}(y)\left|V_{k}^{\delta} \backslash V_{k}^{\delta, \theta}\right|=o(1)
$$

при $\theta \rightarrow 0$.

Из (62), (63) и (64) следует (60). Точно так же доказывается (61). Ясно, что

$$
\int_{V_{k} \backslash V_{k}^{\delta, \theta}} f_{k}(y) d y=\int_{V_{k} \backslash V_{k}^{\delta}} f_{k}(y) d y+\int_{V_{k}^{\delta} \backslash V_{k}^{\delta, \theta}} f_{k}(y) d y,
$$

причем

$$
\int_{V_{k}^{\delta} \backslash V_{k}^{\delta, \theta}} f_{k}(y) d y \leqslant \max _{y \in V_{k}^{\delta}} f_{k}(y)\left|V_{k}^{\delta} \backslash V_{k}^{\delta, \theta}\right| \rightarrow 0, \quad \theta \rightarrow 0 .
$$

Переходя в неравенствах (57) и (58) к пределу по $\theta \rightarrow 0$ и учитывая соотношения (60), (61) (65) и (66), получаем, что

$$
\begin{aligned}
& \limsup _{n \rightarrow \infty} \int_{V_{k}} f_{k}^{(n)}(y) d y \leqslant \sigma^{k} \int_{V_{k}^{\delta}} f_{k}(y) d y+\int_{V_{k} \backslash V_{k}^{\delta}} f_{k}(y) d y, \\
& \liminf _{n \rightarrow \infty} \int_{V_{k}} f_{k}^{(n)}(y) d y \geqslant \sigma^{k} \int_{V_{k}^{\delta}} f_{k}(y) d y .
\end{aligned}
$$

Переходя в этих неравенствах к пределу по $\delta \rightarrow 0$, получаем, что

$$
\begin{aligned}
& \limsup _{n \rightarrow \infty} \int_{V_{k}} f_{k}^{(n)}(y) d y \leqslant \sigma^{k} \int_{V_{k}} f_{k}(y) d y, \\
& \liminf _{n \rightarrow \infty} \int_{V_{k}} f_{k}^{(n)}(y) d y \geqslant \sigma^{k} \int_{V_{k}} f_{k}(y) d y .
\end{aligned}
$$

Отсюда следует, что существует предел

$$
\lim _{n \rightarrow \infty} \int_{V_{k}} f_{k}^{(n)}(y) d y=\sigma^{k} \int_{V_{k}} f_{k}(y) d y .
$$

Существование предела

$$
\lim _{n \rightarrow \infty} S_{k}(n)=\sigma^{k} \int_{V_{k}} f_{k}(y) d y
$$

следует из (55), (56) и (67). Однако согласно (3) и определению функции $f_{k}(y)$

$$
\int_{V_{k}} f_{k}(y) d y=J_{\sigma k}(x) .
$$

Соотношение (14) следует из (68), (69) и (18).

Теорема доказана. 
Из приведенных примеров 1-7 в доказательстве нуждается только утверждение примepa 2.

Доказательство утверждения примера 2. В [16] показано, что при $n \rightarrow \infty$

$$
\begin{aligned}
p(n) & \sim \frac{1}{n} \exp \left(\sum_{k \in A} \frac{1}{k}(1-1 / n)^{k}\right) / \Gamma(\sigma) \\
& =\frac{1}{n} \exp (f(1-1 / n)) / \Gamma(\sigma),
\end{aligned}
$$

где

$$
f(s)=\sum_{k \in A} \frac{s^{k}}{k}, \quad s \in[0,1) .
$$

Согласно соотношению (14) работы [38] при $n \rightarrow \infty$ и $\lambda=-\ln (1-1 / n)$

$$
f(1-1 / n)=f(\exp (-\lambda))=\sum_{k \in A(1 / \lambda)} 1 / k-\sigma \gamma+o(1)
$$

где $\gamma$ - постоянная Эйлера и $A(t)=\{k \in A: k \leqslant t\}$ при $t>0$. Заметим, что при $n \in \mathbf{N}$

$$
\frac{1}{n}=1-e^{-\lambda}<\lambda,
$$

откуда следует, что $1 / \lambda<n$. Поэтому

$$
\sum_{k \in A(n)} \frac{1}{k}=\sum_{k \in A(1 / \lambda)} \frac{1}{k}+\sum_{k \in A(n), k>1 / \lambda} \frac{1}{k} .
$$

Стало быть,

$$
\begin{aligned}
0 \leqslant \sum_{k \in A(n)} \frac{1}{k}-\sum_{k \in A(1 / \lambda)} \frac{1}{k} & \leqslant \frac{(n-1 / \lambda+1)}{[1 / \lambda]+1} \\
& \leqslant \lambda(n-1 / \lambda+1)=\lambda n-1+O\left(\frac{1}{n}\right)=O\left(\frac{1}{n}\right)
\end{aligned}
$$

при $n \rightarrow \infty$, так как при $n \rightarrow \infty$

$$
\ln (1-1 / n)=-\frac{1}{n}+O\left(\frac{1}{n^{2}}\right) .
$$

Из (71) и (72) следует, что при $n \rightarrow \infty$

$$
f(1-1 / n)=\sum_{k \in A(n)} 1 / k-\sigma \gamma+o(1) .
$$

Из (70) и (73) получаем, что при $n \rightarrow \infty$

$$
p(n) \sim \frac{1}{n} \exp \left(\sum_{k \in A(n)} \frac{1}{k}\right) e^{-\sigma \gamma} / \Gamma(\sigma)=n^{\sigma-1} L_{1}(n) e^{-\sigma \gamma} / \Gamma(\sigma),
$$


где функщия

$$
L_{1}(n)=\exp \left(\sum_{k \in A(n)} \frac{1}{k}-\sigma \ln n\right)
$$

медленно меняется на бесконечности, поскольку согласно лемме 1 в [38] последовательность $\exp \left(\sum_{k \in A(n)} \frac{1}{k}\right)$ правильно меняется на бесконечности с показателем $\sigma$, если множество $A$ имеет асимптотическую плотность $\sigma>0$.

Утверждение примера 2 доказано.

\section{Список литературы}

1. Болотников Ю. В., Сачков В. Н., Тараканов В. Е., Асимптотическая нормальность некоторых величин, связанных с цикловой структурой случайных подстановок. Матем. сб. (1976) 99, №1, 121-133.

2. Болотников Ю. В., Сачков В. Н., Тараканов В. Е., О некоторых классах случайных величин на циклах случайных подстановок. Матем. сб. (1979) 108, №1, 87-99.

3. Болотников Ю. В., Тараканов В. Е., Предельные распределения в задачах о циклах случайных подстановок. Комбинаторный и асимптотический анализ. Изд-во Красноярского унив., Красноярск, 1977, с. 62-68.

4. Бендер Э. А., Асимптотические методы в теории перечислений. В кн.: Перечислительные задачи комбинаторного анализа. Мир, Москва, 1979, с. 266-310.

5. Владимиров В. С., Завьялов Б. И., Тауберовы теоремы в квантовой теории поля. Итоги науки и техники. Современные проблемы математики (1980) 15, 95-130.

6. Волынец Л. М., Число решений уравнения в симметрической группе. В сб.: Вероятностные прочессы и их приложения. МИЭМ, Москва, 1985, с. 104-109.

7. Волынец Л. М., Число решений уравнения $x^{s}=e$ в симметрической группе. Матем. заметки (1986) 40, 586-589.

8. Волынец Л. М., Пример нестандартной асимптотики числа подстановок с ограничениями на длины циклов. В сб.: Вероятностные прочессы и их применения. МИЭМ, Москва, 1989, с. 8590.

9. Гончаров В. Л., Из области комбинаторики. Изв. АН СССР, сер. матем. (1944) 8, 3-48.

10. Grusho A. A., Properties of random permutations with constraints on the maximum cycle length. In: Probabilistic Methods in Discrete Mathematics. TVP/VSP, Moscow/Utrecht, 1993, pp. 459-469.

11. Ewens W. J., The sampling theory of selectively neutral alleles. Theor. Popul. Biol. (1972) 3, 87-112.

12. Ивченко Г. И., Медведев Ю. И., О случайных подстановках. Труды по дискретной математике (2002) 5, 73-92.

13. Ивченко Г. И., Медведев Ю. И., Метод В. Л. Гончарова и его развитие в анализе различных моделей случайных подстановок. Теория вероятностей и ее применения (2002) 47, №3, 558-566.

14. Колчин А. В., Уравнения, содержащие неизвестную подстановку. Дискретная математика (1994) 12, №1, 100-115.

15. Колчин В. Ф., Случайные отображения. Наука, Москва, 1984.

16. Колчин В. Ф., О числе циклов подстановки с ограничениями на длины циклов. Дискретная математика (1989) 1, №2, 97-109.

17. Kolchin V. F., The number of permutations with cycle lengths from a fixed set. In: Random Graphs, v. 2. Wiley, Chichester, 1992, pp. 139-149. 
18. Колчин В. Ф., Случайные графы. Физматлит, Москва, 2000.

19. Manstavicius E., On random permutations without cycles of some lengths. Period. Math. Hung. (2001) 42, 37-44.

20. Минеев М. П., Павлов А. И., О числе подстановок специального вида. Матем. сб. (1976) 99, №3, 421-429.

21. Минеев М. П., Павлов А. И., Об уравнении в подстановках. Труды Матем. ин-та им. В. А. Стеклова АН СССР (1976) 142, 182-294.

22. Павлов А. И., О числе циклов и цикловой структуре подстановок некоторых классов. Матем. cб. (1984) 124, №4, 536-556.

23. Павлов А. И., О некоторых классах подстановок с теоретико-числовыми ограничениями на длины циклов. Матем. сб. (1986) 129, №2, 252-263.

24. Павлов А. И., О подстановках с длинами циклов из заданного множества. Теория вероятностей и ее применения (1986) 31, 618-619.

25. Павлов А. И., О числе подстановок с длинами циклов из заданного множества. Дискретная математика (1991) 3, №3, 109-123.

26. Павлов А. И., О числе подстановок с конечными множеством длин циклов. Труды Матем. ин-та им. В. А. Стеклова РАН (1994) 207, 256-267.

27. Павлов А. И., Асимптотика числа подстановок с теоретико-числовыми ограничениями на длины циклов. Докл. РАН (1994) 335, №5, 556-559.

28. Павлов А. И., О двух классах подстановок с теоретико-числовыми ограничениями на длины циклов. Матем. заметки (1997) 62, №6, 881-891.

29. Постников А. Г., Введение в аналитическую теорию чисел. Наука, Москва, 1971.

30. Сачков В. Н., Отображения конечного множества с ограничениями на контуры и высоту. Теория вероятностей и ее применения (1972) 17, №4, 679-694.

31. Сачков В. Н., Случайные отображения ограниченной высоты. Теория вероятностей и ее применения (1973) 18, №1, 122-132.

32. Сачков В. Н., Вероятностные методы в комбинаторном анализе. Наука, Москва, 1978.

33. Сенета Е., Правильно меняющиеся функции. Наука, Москва, 1985.

34. Тараканов В. Е., Чистяков В. П., О цикловой структуре случайных подстановок. Матем. сб. (1975) 96, №4, 594-600.

35. Shepp L. A., Lloyd S. P., Ordered cycle lengths in a random permutation. Trans. Amer. Math. Soc. (1966) 121, №2, 340-357.

36. Феллер В., Введение в теорию вероятностей и ее приложения, т. 2. Мир, Москва, 1984.

37. Якымив А. Л., О числе А-подстановок. Матем. сб. (1989) 180, №2, 294-303.

38. Якымив А. Л., О подстановках с длинами циклов из заданного множества. Дискретная математика (1989) 1, №1, 105-116.

39. Якымив А. Л., О случайных подстановках с длинами циклов из заданного множества. В сб.: Вероятностные прочессы и их приложения. МИЭМ, Москва, 1991, с. 24-27.

40. Якымив А. Л., О некоторых классах подстановок с длинами циклов из заданного множества. Дискретная математика (1992) 3, №3, 128-134.

41. Якымив А. Л., О числе подстановок с длинами циклов из случайного множества. Дискретная математика (2000) 12, №4, 53-62.

42. Yakymiv A. L., Limit theorems for random A-permutations. In: Probabilistic Methods in Discrete Mathematics. TVP/VSP, Moscow/Utrecht, 1993, pp. 459-469. 